\title{
A Review on Evolution of 3GPP Systems Interworking with WLAN
}

\author{
Rajavelsamy $\mathrm{R}^{1}$, Manoj Choudhary ${ }^{1}$ and Debabrata Das ${ }^{2}$ \\ ${ }^{1}$ Samsung R\&D Institute India - Bangalore (SRI-B), Bangalore \\ ${ }^{2}$ International Institute of Information Technology - Bangalore (IIITB), Bangalore \\ E-mail: \{rajvel, choudhary.m\}@samsung.com; ddas@iiitb.ac.in
}

Received 3 March 2016;

Accepted: 19 March 2016

\begin{abstract}
Evolution of Radio Access Technologies (RATs) is succeeding on its fifth generation $(5 \mathrm{G})$. Over the past decade, interworking between heterogeneous RATs has undergone remarkable growth, as there is explosive growth in mobile devices such as smartphones supporting multiple RATs, especially Global System for Mobile communications (GSM), Code division multiple access (CDMA), Universal Mobile Telecommunications System (UMTS), High Speed Packet Access (HSPA), Long Term Evolution (LTE), Wireless Local Area Network (WLAN). WLAN is complementary options available to mobile operators when they consider expanding the user data traffic capacity further from the unlicensed spectrum. Since 2004 (Release-6), the Third Generation Partnership Project (3GPP) [1] has initiated to develop a 3GPPWLAN interworking architecture to allow 3GPP service providers to offload data traffic from wide area wireless spectrum to WLANs in indoor locations, hotspots, and other areas with high user density. The initial work in 3GPP specified loose integration (at the core network) and progressively introduced potential enhancements for tighter integration (at the radio access network) based on market trends and demands. This paper provides an insight on the evolution of 3GPP systems interworking with WLAN from 3GPP Release-6 to Release-13.
\end{abstract}

Journal of ICT, Vol. 3, 133-156.

doi: $10.13052 /$ jicts2245-800X.322

(c) 2016 River Publishers. All rights reserved. 


\section{Introduction}

WLAN represents an efficient approach to address the capacity, spectrum limitations and effective indoor coverage. Indeed, WLAN presents complementary characteristics with 3GPP networks which motivate their interworking. One of the key requirements in next generation mobile networks is to make use of unlicensed spectrum by efficient interworking with WLAN. WLAN is the most widely deployed technology operating in the unlicensed radio band, which covers indoors and can therefore be used as a free license radio link. Multi-RAT devices (e.g. HSPA, LTE, Bluetooth and WLAN) are becoming commonly available and the applications running in the mobile devices are expanding. In some environments (e.g. home, enterprise, and public places) it would be beneficial for operators to offload certain type of traffic from $3 \mathrm{GPP}$ radio to WLAN. Interworking mechanisms in 3GPP initially addressed the smart traffic steering between the heterogeneous RATs using classical Internet Protocol (IP) routing functionalities till Release-11 and then started to address radio level aggregation in order to overcome from under-utilization of operator deployed WLAN, unnecessary WLAN scanning and overloading the WLAN networks.

3GPP Release- 6 work was on interworking between the WLAN and the 3rd Generation (3G) UMTS core network (I-WLAN) [2]. In Release-6, 3GPP addressed the definitions of various interworking use cases and specified architectures with different interworking scenarios [2]. Interworking scenarios directed to different architecture implementations alternatives, extending from loosely-coupled to tightly-coupled interworking architectures based on the core network entities interconnection between the mobile network and the WLAN [3].

In the 3GPP Release-7 specifications, Private Network access from I-WLAN was addressed. A mechanism to perform authentication between the WLAN UE and an AAA server located in an external Packet Data Network (PDN) was specified, in addition to I-WLAN access authentication. Further, enhancements to support Quality of Service (QoS) provisioning over I-WLAN were also addressed.

In the 3GPP Release-8, integration work was carried to the 3GPP defined Evolved Packet Core (EPC) for LTE-WLAN interworking [4]. 3GPP specified the description for providing IP connectivity using non-3GPP accesses to the evolved Packet Switched (PS) domain [5]. In the 3GPP Release-8 specification on interworking between the WLAN and the EPC, 3GPP addressed all interworking aspects, including mobility between $3 \mathrm{GPP}$ and non-3GPP accesses, 
security aspects (authentication, secure communication), policy control and charging, related to the usage of non-3GPP accesses. 3GPP Release- 8 does not allow simultaneous connections to multiple access networks [5]. Further, the 3GPP defined Access Network Discovery and Selection Function (ANDSF) framework for non-roaming scenarios to provide access network information to the UE, to enhance the discovery of non-3GPP access networks by the UE.

In Release-9, the ANDSF was enhanced to provide access network discovery and selection information for both roaming and non-roaming scenarios. This information is delivered to the UE in a secure manner. In addition, network discovery and selection functionality was optimized, so it offers minimal signalling overhead and supports service continuity between 3GPP and non-3GPP access systems [6].

In the 3GPP Release-10, simultaneous network connections to multiple radio access technologies were specified [5]. 3GPP specified the mechanism to enable both seamless and non-seamless IP flow mobility between a 3GPP and WLAN radio access networks. Seamless offload indicates the capability to seamlessly move one or more selected IP flows between the 3GPP network and non-3GPP access network (WLAN), while providing IP session continuity. Non-seamless offload indicates the possibility to exchange the traffic of one or more selected IP flows using WLAN IP address (referred also as Scenario-2 Direct IP Access in I-WLAN) without providing any IP session continuity. Seamless offload is achieved using Dual-stack Mobile IPv6 (DSMIPv6) and Proxy Mobile IPv6 (PMIPv6) IP mobility protocols in the EPC. 3GPP defined the capability for DSMIPv6 capable UEs to allow seamless offload of individual IP flows corresponding to a PDN connection from EPC to WLAN by introducing IP flow mobility (IFOM) support to the EPC. The IFOM features allows mobile operators to dynamically direct individual IP flows which may belong to the same PDN connection to specific access RAT via the DSMIPv6 mobility solution. Further, Network-based mobility between Evolved Universal Terrestrial Radio Access Network (E-UTRAN) and untrusted non-3GPP accesses is provided using PMIPv6 protocol. 3GPP System interworking with fixed broadband access network was specified in Release-11.

Till 3GPP Release-12, mechanisms have been defined for simultaneous connectivity over 3GPP and WLAN for different PDN connections and for Non-Seamless WLAN Offload (NSWO). However, as operator controlled WLAN deployments become more common and WLAN usage increases, 3GPP identified that RAN level enhancements are essential for WLAN interworking to improve user experience, provide more operator control, 
for better access network utilization and to reduced operational expenditure (OPEX). 3GPP focused on Radio Access Network (RAN) level integration between LTE and 3GPP operator controlled WLAN (or their partners) with the objective to enable coordinated radio resource management between the LTE and the WLAN. 3GPP specified the mechanisms on Radio Access Network (RAN) level integration between the LTE and operator controlled WLAN and introduced RAN rules and assistance information which help in making better traffic steering decisions, with more RAN control [5].

In 3GPP Release-13, work is on progress, which aimed at improving LTE-WLAN Radio Level Aggregation and Integration [7].

The rest of the paper is organized as follows: Section 2 provides the insight on 3G-WLAN interworking system, as specified by 3GPP in Release-6 and in Release-7. In Section 3 we provide the insight on LTE-WLAN interworking system, as specified by 3GPP in Release-8 till Release-9. In Section 4, details of LTE-Advance-WLAN interworking mechanism is specified. Section 5 provides the insight on RAN level interworking, as specified by 3GPP in Release-12. 3GPP specified Release-13 mechanism for LTE-WLAN aggregation is described in Section 6. We summarise the paper in Section 7.

\section{3G-WLAN Evolution}

\subsection{Release-6}

\subsubsection{G-WLAN interworking scenarios}

3GPP specified an approach for a flexible, general, scalable and future proof 3G-WLAN interworking [3]. The specified approach can be implemented in steps from a quite simple 3G-WLAN interworking, to fully seamless inter system operation. Specified approach defines six different scenarios for interworking that provide an indicative long term goals for development. Each scenario realizes an additional step in integrating WLAN in the 3GPP service offering and naturally includes the previous level of integration of the previous scenario as shown in Table 1, for step by step progressive deployment.

Scenario 1 - Common billing and customer care: This provides only a common bill to the subscriber.

Scenario 2 - 3GPP system-based access control and charging: This scenario requires authentication, authorization, and accounting (AAA) for 3GPP subscribers in the WLAN to be based on the same subscription credentials utilized in the 3GPP system for WLAN access also to enable IP connectivity. 
Table 1 3GPP specified scenarios and corresponding features

\begin{tabular}{|c|c|}
\hline \multicolumn{2}{|l|}{ Interworking } \\
\hline Scenarios & Supported Features \\
\hline 1 & Common billing and customer care \\
\hline 2 & Scenario 1 and $3 \mathrm{G}$-based access control and charging \\
\hline 3 & $\begin{array}{l}\text { Scenario } 2 \text { and access to } 3 \mathrm{G} \text { packet-switched based } \\
\text { services }\end{array}$ \\
\hline 4 & Scenario 3 and service continuity \\
\hline 5 & Scenario 4 and seamless service continuity \\
\hline 6 & $\begin{array}{l}\text { Scenario } 5 \text { and access to } 3 \mathrm{G} \text { circuit-switched based } \\
\text { services with seamless mobility }\end{array}$ \\
\hline
\end{tabular}

Scenario 3 -Access to 3GPP system packet-switched (PS) based services: This scenario is to allow the mobile operator to spread out access to $3 \mathrm{G}$ services to WLAN access network. In general, no service continuity across the access networks is required in Scenario 3.

Scenario 4 - Service continuity: To allow access to 3G-based services as required by Scenario 3, and in addition to maintain service continuity across the $3 \mathrm{G}$ and WLAN systems. The service continuity requirements for Scenario 4 are not very stringent, which means service may be terminated when moved in to a WLAN system or change in service quality may be possible.

Scenario 5 - Seamless services: One step further more than Scenario 4, to provide seamless service continuity between $3 \mathrm{G}$ and WLAN. That is, $3 \mathrm{G}-$ based services should be utilized across the 2G/3G and WLAN access technologies in a seamless manner.

Scenario 6-Access to 3GPP circuit-switched (CS) services: This scenario is to offer access to CS services from the WLAN access network.

\subsubsection{G-WLAN interworking architecture}

3G-WLAN interworking architecture, as specified by 3GPP [3], is focused on the interworking functionality between 3GPP based UMTS core network and 802.11 technology based WLAN systems. The intent of 3G-WLAN interworking is to extend $3 \mathrm{G}$ services and functionalities to the WLAN access environment [8].

The IEEE 802.11 WLAN effectively becomes a complementary radio access technology to the $3 \mathrm{G}$ system. The typical 3G-WLAN interworking System, as specified in 3GPP is shown in the Figure 1. The User Equipment (UE), as referred in this paper, is a combination of ME (Mobile Equipment) with SIM/USIM (Subscriber Identity Module/UMTS Subscriber Identity Module) and WLAN UE. The 3G-WLAN interworking requires the ability for 


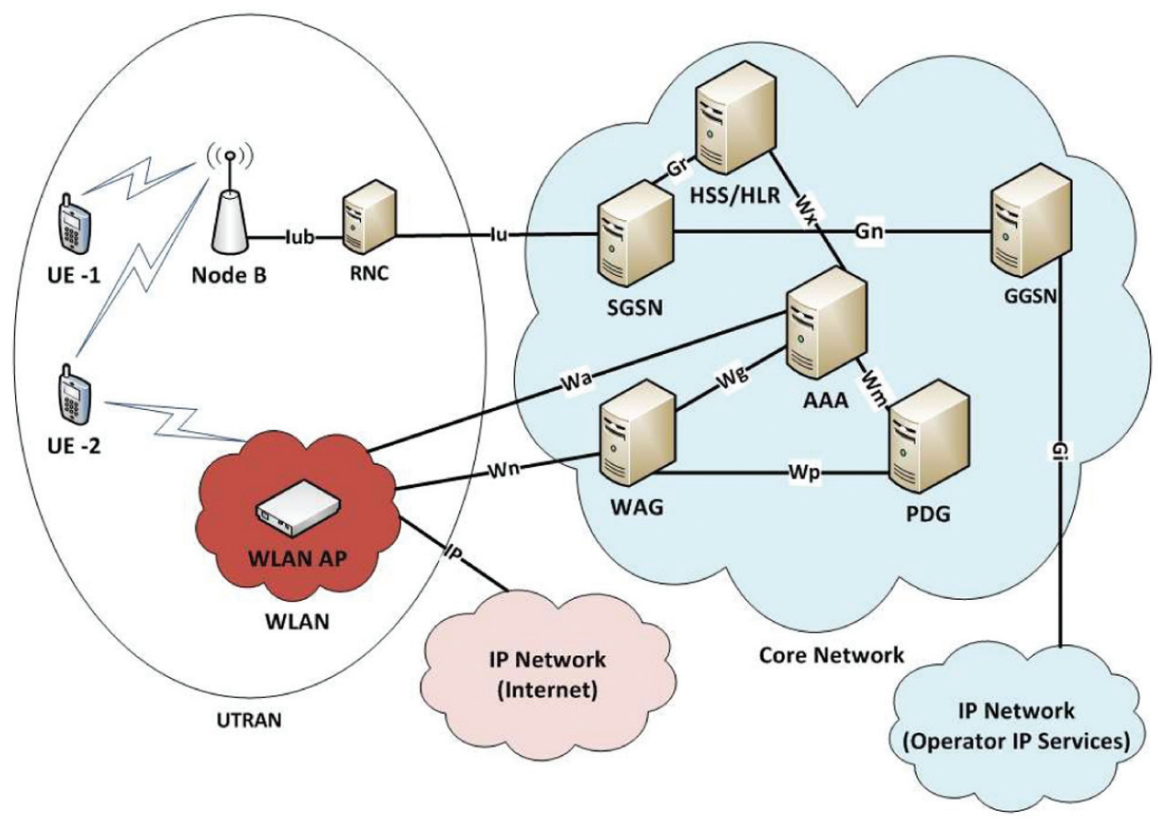

Figure 13 G-WLAN interworking system.

a SIM/USIM to be used for providing common access control and charging for WLAN and 3G services using the 3GPP specified system infrastructure. The WLANs infrastructure mode network architecture resembles the widearea mobile networks and is of most interest to 3GPP in defining 3G-WLAN interworking.

The packet transmission over the air between the WLAN Access Point (AP) and the UE is protected by Wired Equivalency Privacy (WEP). The 802.11i [2] standard is used to overcome limitations on confidentiality, integrity, and access control in the 802.11 networks.

\subsubsection{G service access through WLAN}

To access $3 \mathrm{G}$ services, the subscriber needs to first authenticate and get authorized from the 3GPP network, and then WLAN access network allows the UE to access the $3 \mathrm{G}$ Core network. An end to end tunnel is then set up between a UE and a PDG to direct all UE packets through the $3 \mathrm{G}$ operator network (Scenario 3). Some 3G services are provided only when the UE access the $3 \mathrm{G}$ core network. Examples of such services include the Multimedia Messaging Service (MMS) and Wireless Application Protocol (WAP). According to the 
3GPP specification [8], Internet Protocol Security (IPsec) [9] tunnel using Encapsulated Security Protocol (ESP) [10] is used between the UE (within WLAN AN) and the PDG of $3 \mathrm{G}$ core network. To access $3 \mathrm{G}$ services from WLAN, a UE uses two IP addresses namely a Local IP address and a Remote IP address. The Local IP address is to deliver the IP packet to a UE in a WLAN AN and identifies the UE in the WLAN. The Remote IP address is used to transport the encapsulated data packet over the IPSec tunnel. It represents the identity of the UE in the network and is used by UE in accessing the $3 \mathrm{G}$ service. The Remote IP address is used for the inner packet (containing encapsulated data payload) of the IPSec tunnel. Figure 2 shows the IPSec Tunnel Mode encapsulation.

\subsection{Release-7}

\subsubsection{QoS support for 3G-WLAN interworking}

When using 3GPP IP Access, a tunnel from UE to PDG is established for carrying PS based services traffic (I-WLAN Scenario 3) [11]. It is possible

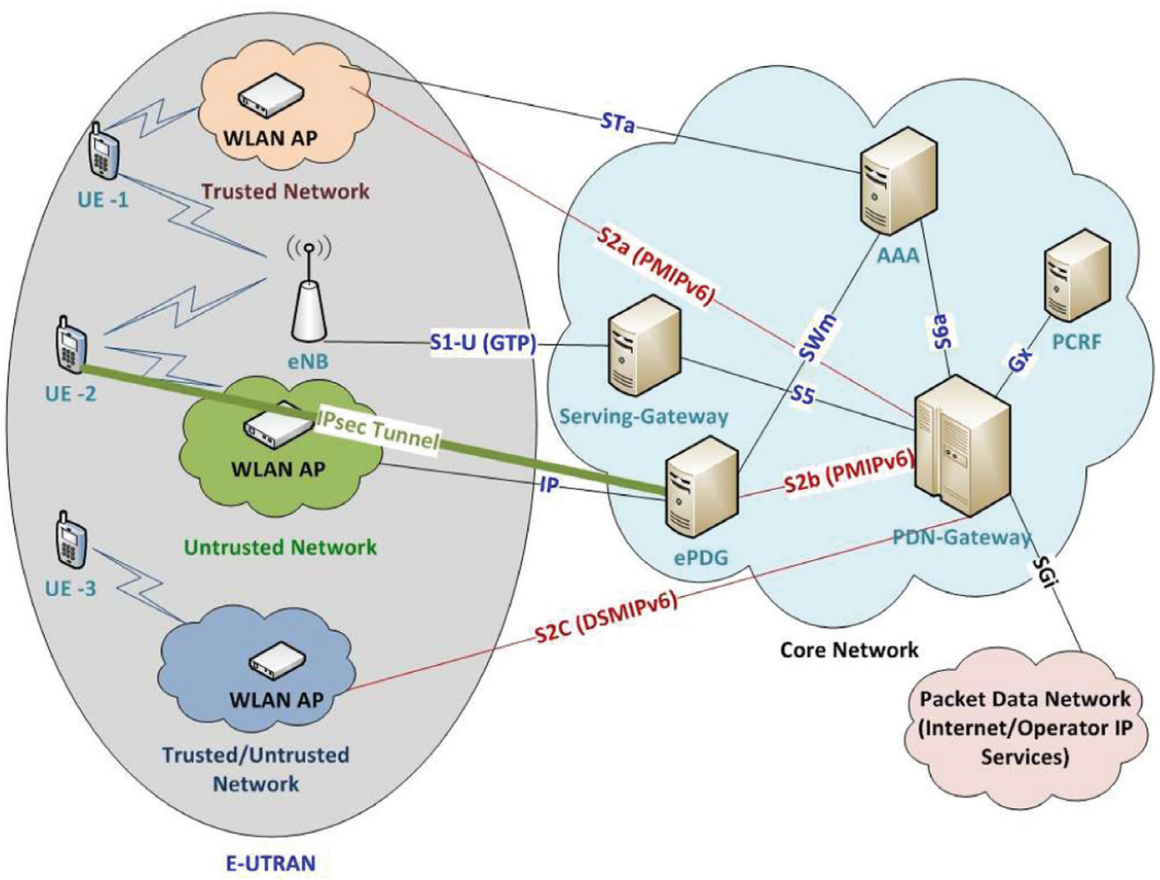

Figure 2 LTE-WLAN interworking system. 
that the user data for different services (more than one IP flow) are carried in one IPsec tunnel between the UE and the PDG; therefore, it may not be possible to separate out individual IP flows of a particular service at intermediate nodes.

For this scenario, 3GPP specified to use DiffServ between the UE and the PDG to appropriately colour the DS Field in the external IP header of the IPsec tunnel to provide different levels of QoS for different classes of service [3].

\subsubsection{Private network access from WLAN 3GPP IP access}

3GPP specified the support for Password Authentication Protocol (PAP) and Challenge-Handshake Authentication Protocol (CHAP) over Extensible Authentication Protocol (EAP) for the authentication and authorization for the Private network access. 3GPP recommended CHAP over PAP, as PAP is an obsolete protocol [12].

\section{LTE-WLAN Evolution}

\subsection{Release-8}

\subsubsection{EPC-WLAN interworking architecture}

3GPP specified as part of Evolved Packet Core (EPC), the support of connectivity over non-3GPP networks (WLAN) and also transfer of IP traffic between 3GPP access and non-3GPP technologies [4]. As shown in Figure 2, access to the EPC is supported via non-3GPP access, such as WLAN technologies [5] and via 3GPP access, such as LTE (E-UTRAN, via evolved NodeB (eNB) [24]). All non-3GPP-specific access technologies are typically connected through the Packet Data Network (PDN) gateway (PDN-GW) or through the evolved Packet Data Gateway (ePDG), which provides extra security functionality for untrusted access technologies. For access to the EPC through the WLAN different new interfaces are specified. A WLAN is directly connected to a PDN-GW through the S2a interface as trusted access. On the other hand, a WLAN is accessible through the ePDG as a non-trusted access. All user data paths from the 3GPP and non-3GPP access networks are combined at the PDN-GW, which includes functionality such as packet filtering, legal lawful interception, generating charging records, IP address allocation (PDN based) and routes traffic over SGi to an external PDN or to the operator's packet data network (for accessing IP services provided by the operator). Apart from the network entities handling the user plane 
data traffic, the EPC also contains network control entities for keeping user subscription information (Home Subscriber Server - HSS), determining the identity and privileges of a user and tracking the activities (AAA server), and enforcing charging and QoS policies through a Policy and Charging Rules Function (PCRF).

IP access procedures to the non-3GPP differ based on whether the non3GPP access system is Trusted or Untrusted. Whether a non-3GPP IP access network is Trusted or Untrusted is not a characteristic of the access network, it is the 3GPP operator's decision [13], who administrates the EPC which interworks with the non-3GPP networks. The 3GPP operator's decision can be based on the non-3GPP access network deployment scenarios and security features provided/supported by the non-3GPP access network are considered sufficiently secure or not sufficiently secure by the 3GPP operator. 3GPP network provides indication whether the non-3GPP IP access is trusted or not, to the UE to initiate appropriate procedures [5] with the non-3GPP access network.

3 GPP enhanced the functionality of the PDG (specified for I-WLAN) to support interworking with EPC via an untrusted access and termed as Evolved Packet Data Gateway (ePDG). The UE has to establish an IPsec tunnel with the ePDG to access operator's services. In addition to the functionality defined for the PDG in 3GPPTechnical Specification (TS) 23.234 [3] for the allocation of a remote IP address, ePDG supports the following functionalities: routing of packets between the PDN GW and the UE, Mobile Access Gateway (MAG) if network based mobility is used, Local Mobility Anchor (LMA) within untrusted non-3GPP access networks using MOBIKE (if needed), enforcement of QoS policies based on information received via AAA infrastructure. 3GPP specified multiple mobility mechanisms for legacy 3GPP and LTE accesses to interwork with non-3GPP accesses which can in general be classified into client based mobility mechanisms and network based mobility mechanisms. With the introduction of ePDG, the UE when performing trusted access network connects directly to the PDN-GW, while the UE performing nontrusted network connects to the PDN-GW through the ePDG as shown in Figure 2.

\subsubsection{EPC-WLAN mobilty procedures}

3GPP identified that it is beneficial to provide mobility support (i.e. preservation of IP address) while UE's move between the 3GPP access networks and the WLAN [14]. 3GPP specified mobility of all packet connections between 3GPP access and non-3GPP access are classified into two categories: optimized and non-optimized handover. Non-optimized handover covers a 
situation where the source network is not involved in preparing resources in the target network (for example, between the LTE and the WLAN). In the case of optimized handovers, the source network is involved in preparing resources in the target network (for example, between the LTE and the CDMA). During mobility, UE's IP addresses are preserved and all the PDN connections in an access network are handed over to other access network. 3GPP specification allows two IP mobility management mechanisms, Network based Mobility and UE based Mobility, for non-3GPP access. 3GPP specified the following interfaces for seamless mobility [5]:

$S 2 a$ : is for network based mobility, based on PMIPv6, to enable access via Trusted non-3GPP IP accesses. S2a provides the user plane with related control and mobility support between trusted non-3GPP IP access and the PDN Gateway.

$S 2 b$ : is also for network based mobility based on PMIPv6, to enable access via Untrusted non-3GPP IP accesses. S2b provides the user plane with related control and mobility support between ePDG and the PDN Gateway.

$S 2 c$ : is for UE based mobility mechanisms, based on DSMIPv6, to enable access via both Trusted/Untrusted non-3GPP IP accesses. S2c provides the user plane with related control and mobility support between the UE and the PDN Gateway.

\subsubsection{Access network discovery and selection function (ANDSF)}

3GPP defined ANDSF as an optional network element located in the operator's core network (EPC), for policy provisioning to the UE in order to perform appropriate traffic steering decisions between a 3GPP access network and non-3GPP access network [5]. The ANDSF provide UE's with network information and operator-defined policies to escort network selection decisions. A new standardized interface (S14) is used between the ANDSF and the UE to distribute the network selection information and policies, as shown in Figure 3 [5]. The procedures defined in the Release- 8 for WLAN access selection and PLMN selection replaces the corresponding I-WLAN procedures specified in pre-Release- 8 specifications.

\subsection{Release-9}

\subsubsection{ANDSF enhancements}

3GPP specified the principles and requirements to be applied for roaming scenarios that is when the UE is registered in a Visited PLMN (VPLMN), when both $3 \mathrm{GPP}$ and non-3GPP accesses are available or when multiple 


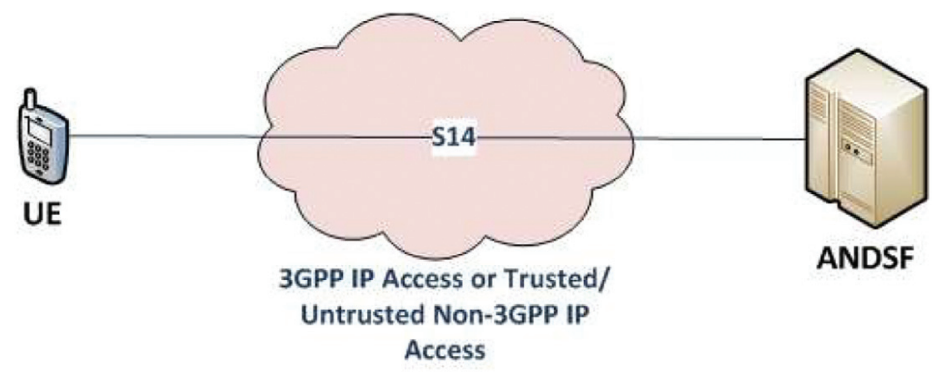

Figure 3 S14 interface for ANDSF.

non-3GPP accesses are available. In case the UE interacts with both H-ANDSF and V-ANDSF, then the UE required to resolve potential conflicts between the ANDSF information received by the two ANDSFs. In such cases, 3GPP decided that the ANDSF policies provided by the VPLMN were allowed to prevail for the decisions.

\section{LTE Advance (LTE-A)-WLAN Evolution}

\subsection{Release-10}

LTE-Advanced promises higher data rates than 3GPP Release- 8 or Release-9 systems and since it is required to be defined on top of existing architectures, requiring no or minimal enhancements to deploy. 3GPP addressed the higher data rates of LTE-A by enabling simultaneous network connections to multiple radio access technologies, using both Seamless WLAN offloading and Nonseamless WLAN offloading (NSWO). When the UE is under WLAN coverage, it is beneficial to offload best effort to the WLAN access and to keep QoS stringent applications like VoIP call in the $3 \mathrm{GPP}$ access network. Non-seamless WLAN offload is achieved by acquiring a local IP address on WLAN by the UE, particular IP flows are routed via the WLAN without pass through the 3GPP core network (similar to I-WLAN Scenario 2) and no IP address preservation in the EPC is provided between WLAN and 3GPP accesses for mobility. 3GPP specifications allows for a UE which also supports seamless WLAN offload to perform seamless WLAN offload for some selected IP flows and non-seamless WLAN offload for some other selected IP flows simultaneously [15]. 3GPP realized seamless WLAN offloading mechanisms using Multi Access PDN Connectivity (MAPCON) and IP Flow Mobility (IFOM), which are detailed in the following sections. 


\subsubsection{Multi access PDN connectivity (MAPCON)}

MAPCON [15] offers the UEs to establish multiple connections to different PDNs via different access networks and also a selective transfer of PDN connections between access networks. The usage of multiple PDNs is typically controlled by the network operator's policies. By using MAPCON, offloading can be achieved relatively easily without requiring the UE to support multiple client-based mobility protocols like DSMIPv6. The Access Point Names (APNs) used over different accesses must be distinct in MAPCON and also if there is more than one PDN connection to a single APN, then all the PDN connections which belong to an APN must be over the same access network.

\subsubsection{IP flow mobility (IFOM)}

IFOM [15] allows the operator to use WLAN as a seamless extension to 3GPP access network and to increase the overall system capacity. IFOM is a mobility management method that connects the UE to the 3GPP and to the non-3GPP networks to the same PDN (session with a PDN-GW) and maintains the IP

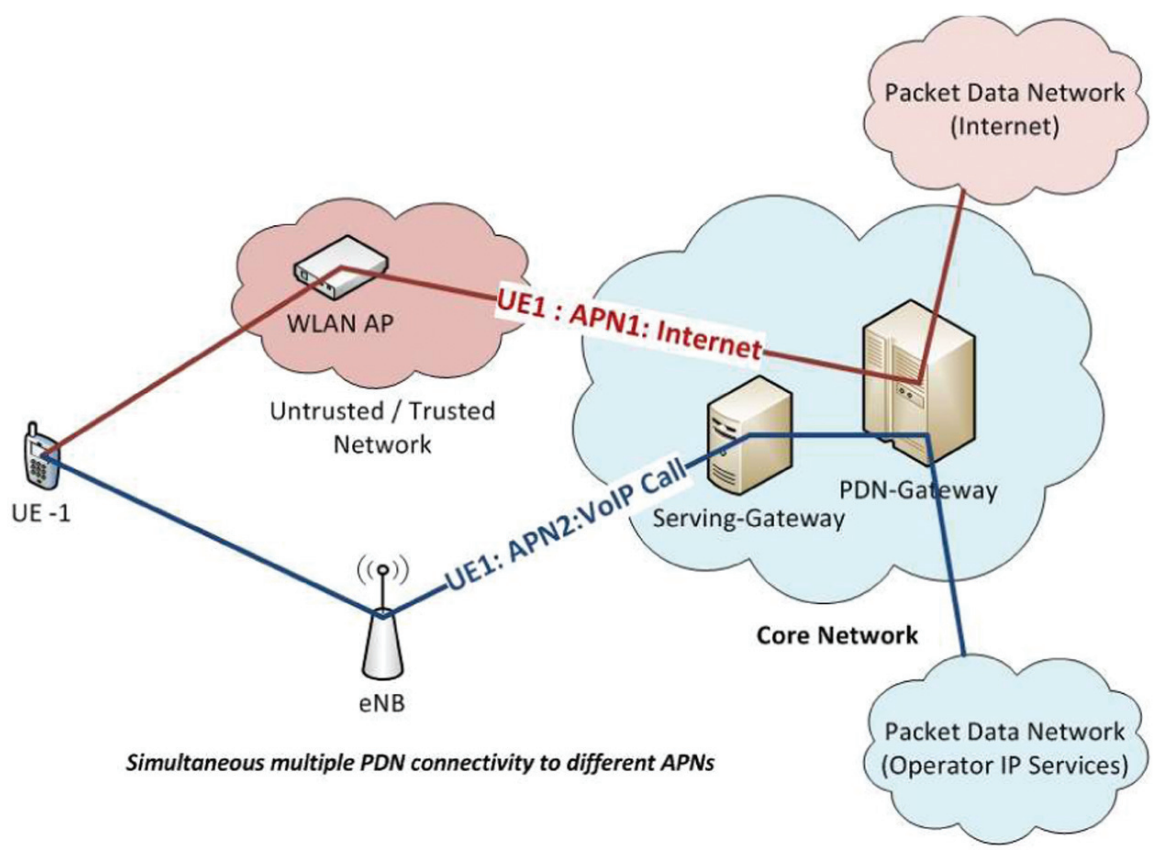

Figure 4 Multi access PDN connectivity. 
connectivity using DSMIPv6. In the case of IP flow mobility, the same APN is configured simultaneously over LTE and WLAN access networks. In the same way as MAPCON, downloading large files using FTP is performed via the WLAN network while voice calls using Voice over LTE (VoLTE) and video calls are handled via the 3GPP network. The logic to offload data is more UE centric in IFOM and basically transparent to the RAN. At mobility between 3GPP and WLAN access, the UE determines which IP flows of a PDN connection are offloaded. For example, best effort traffic is offloaded from the 3GPP to the WLAN access (as soon as WLAN is in the proximity), while video streaming flow of the same APN remains on the 3GPP access network.

\subsubsection{ANDSF enhancements}

The ANSDF is further enhanced in the Release-10 to provide inter-system routing policies for any given APN to be used on the UE. ANSDF steers traffic in the case of non-seamless 3GPP offloading, multiple access PDN connections, and IP flow mobility. The 3GPP defines the use of ANSDF policies for the DSMIPv6 solution by matching a given traffic selector on a given access network.

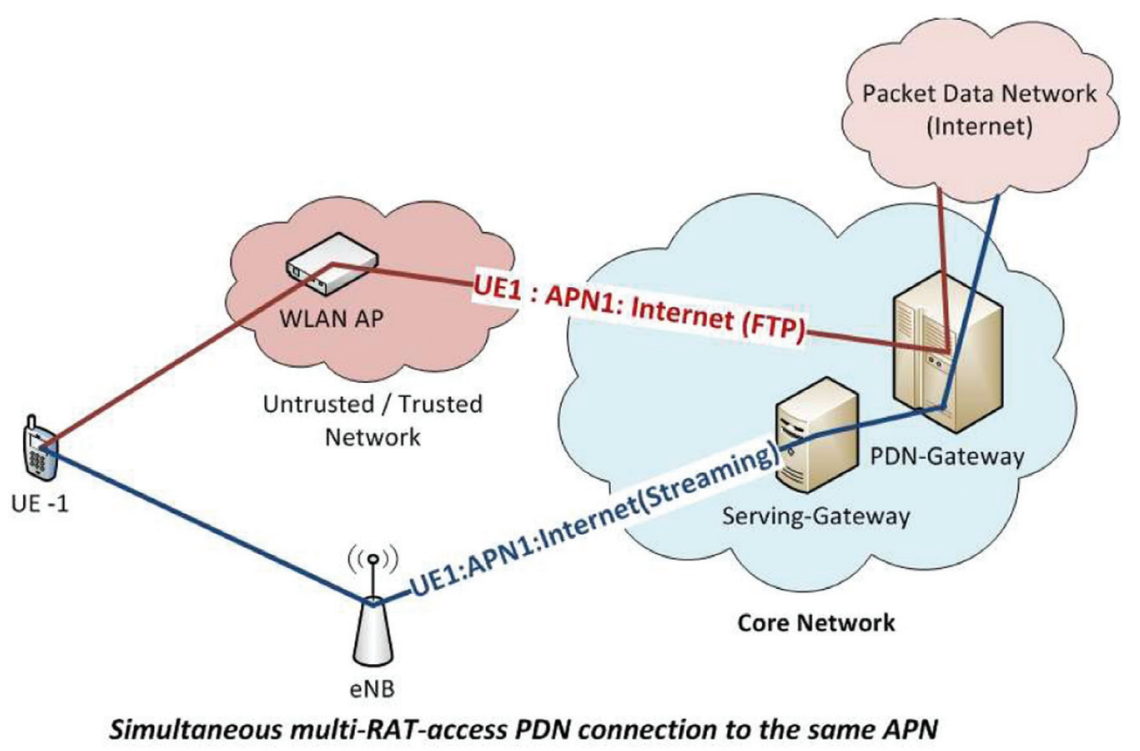

Figure 5 IP flow mobility. 


\subsection{Release-11}

In the Release-11, 3GPP focused on Fixed-Mobile Convergence (FMC) and specified system description (covering mobility and QoS aspects) for the support of BroadBand Forum Accesses Interworking (BBAI) [21]. Interworking between the 3GPP system and the Fixed Broadband Access network defined by Broadband Forum (BBF) is to provide the IP connectivity to a 3GPP UE using a WLAN connected to a Fixed Broadband Access network. The interworking architecture for BBAI is based on the EPC interworking with non-3GPP access network architecture defined in TS 23.402 [5].

Further in the Release-11, based on the requirements from the operators to use GPRS Tunnelling Protocol (GTP) based S2a for WLAN to access EPC, 3GPP supported GTPv2 over S2a, under S2a Mobility based on GTP (SaMOG) work item. The use of GTP based S2a or PMIPv6 based S2a for the WLAN to access the EPC depends on the operator's network deployment policy [5].

\section{LTE-WLAN RAN Level Interworking}

\subsection{Release-12}

\subsubsection{RAN-assistance information}

The 3GPP has closely investigated the access thresholds for offloading decisions and decided to extend ANDSF policies to enhance 3GPP-WLAN interworking at radio level. The enhancement includes within the access network selection policies a subset of information provided by Hotspot 2.0 Release-2 [16] which is considered to be relevant to 3GPP interworking scenarios. As pre-release-12 ANDSF based information for access network selection and traffic routing does not consider either the RAN network conditions or the factors such as the WLAN load conditions. Also the ANDSF does not provide mechanisms to indicate preferences with granularity at the 3GPP RAT level within network policies. This restricts the ability for the operator to provide policies that favour a specific $3 \mathrm{GPP}$ RAT over another one with reference to the WLAN preference.

The objective of indicating the preference with granularity at the 3GPP RAT level is to use the evolved NodeB (eNB) initiative to steer the UE traffic onto either the operator controlled WLAN or the LTE, depending on the requisite. Release-12 specifies a mechanism for 3GPP-WLAN access network selection and traffic steering based on RAN-assistance information, as only 
RAN has a comprehensive overview of its load conditions and resource allocation strategies. The Release- 12 solution supports deployments both with and without ANDSF and the co-existence of ANDSF with RAN rules, when both are deployed, as shown in Figure 6. The RAN Assistance Parameters (RAP) includes the following thresholds and indication, 3GPP access thresholds, WLAN access thresholds and an Offload Preference Indication (OPI) value. In a network without enhanced ANDSF deployment or with UEs without ANDSF support, these RAP are included within the RAN rules. In networks that support ANDSF and which have UEs capable of ANDSF, the RAP are used as part of the ANDSF policies. The WLAN access selection and the traffic routing behaviour of a UE is controlled either by the ANDSF rules or by the RAN rules, not by both. In the defined Release-12 mechanism, the RAP are broadcasted via System Information Blocks (SIB) and/or via dedicated signalling. To decide on the offloading for the UEs in the Radio Resource Control (RRC) CONNECTED state, the network use the dedicated traffic steering commands, based on WLAN measurements reported by the UE.

Even though 3GPP has specified significant enhancements on the ANDSF network and discovery functionalities till Release-11, the newly specified WLAN network and Service Provider selection mechanism supersedes the pre-Release-12 WLAN network and PLMN selection feature. Based on

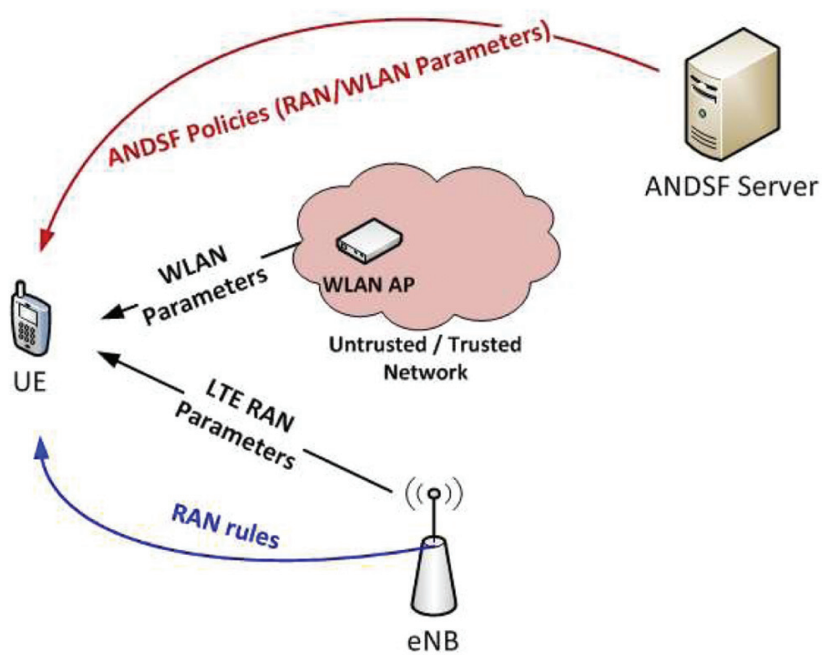

Figure 6 Traffic steering using RAN-assistance information. 
Release-12 RAP extensions, 3GPP decided to stop maintaining the I-WLAN solutions, as the ANDSF/RAN-based solution that is specified in Release-12 provides an alternative and more up-to-date solution for WLAN network selection than the I-WLAN network selection.

\subsubsection{Enhanced interworking}

3GPP system architecture group revisited the inter-RAT offloading modes and enhanced the S2a solution with three offloading operation modes [5] as below:

- Single-Connection mode (SCM) which only supports NSWO. The SCM supports the IP address preservation between the 3GPP and the WLAN access network in the EPC. SCM mode does not require additional protocols in order to establish NSWO.

- Multi-Connection mode (MCM) which supports simultaneously one or more PDN connections and/or NSWO over trusted WLAN access. The MCM supports also the IP address preservation between the 3GPP and the trusted WLAN access network in the EPC. The MCM uses a new specific protocol between the UE and the trusted WLAN access network called WLAN Control Protocol (WLCP) to trigger the establishment and release of PDN connectivity from the trusted WLAN access network [5].

- Transparent Single-Connection mode in which handover-indicator from the UE, APN indication from the UE and the Protocol Configuration Options (PCO) via WLAN are not supported. As a consequence, handover between trusted WLAN access network and 3GPP access with IP address preservation in EPC is not supported. This operational mode is equivalent to pre-Release-12 functionalities.

The operation mode is negotiated between the UE and the 3GPP network as part of the authentication procedure based on the 3GPP specific extensions to the EAP-AKA' [17] protocol.

\section{LTE-WLAN Carrier Aggregation}

\subsection{Release-13}

The two components considered in the Release-13 are LTE-WLAN Carrier Aggregation and Interworking enhancements. Release-13 focus was on aggregation of LTE and WLAN at Packet Data Convergence Protocol (PDCP) layer. While it is possible to aggregate the LTE-WLAN technologies at higher layers (as specified in pre-Release-13 specifications), performing aggregation 
at the RAN level such as the PDCP layer benefits all applications and gives more control to operators. LTE-WLAN Aggregation introduces user plane data aggregation where a user plane data radio bearer can be served by the LTE and/or by the WLAN. Release-13 solution aims to provide better control and utilization of resources on both radio links and increase the aggregate throughput, system capacity and will be transparent to the core network $(\mathrm{CN})$. The common element of both components is the RAN control of offloading, where the eNB make the traffic steering decision based e.g., on UE measurements reporting, as well as feedback from the WLAN. The solutions were applied to both collocated (integrated eNB and WLAN access point) and non-collocated (eNB and WLAN are connected via backhaul) scenarios.

\subsubsection{LTE-WLAN Aggregation (LWA)}

LTE-WLAN interworking solution till Release-12 enhances CN-based WLAN offloading by providing more control to operators and by improving network utilization. In Release-13, 3GPP further specified enhancements for LTEWLAN interworking by LTE-WLAN carrier aggregation mechanism, for both co-located and non-co-located deployment scenarios. Release-13 LTEWLAN Radio Level Integration provides a RAN level aggregation solution with operator control that can also take radio conditions into account and improves mobility to and from the WLAN thereby improving overall throughput by using both the 3GPP and the WLAN access. The advantages of the LTE-WLAN aggregation are as follows:

- The WLAN access network becomes transparent to $\mathrm{CN}$ in the sense that interworking does not require any WLAN specific $\mathrm{CN}$ nodes and $\mathrm{CN}$ interfaces.

- Radio level carrier aggregation allows for the realtime channel and radio resource management with load aware across the LTE and the WLAN to provide significant improvements in the capacity and Quality of Experience (QoE).

- The LTE network can be used as a control and mobility anchor network to provide QoE improvements, minimize service interruption, and increase operator control.

- No new WLAN-related CN signalling is needed, thus reducing $\mathrm{CN}$ signalling load.

For the non-co-located case, $3 \mathrm{GPP}$ specified an external interface $\left(\mathrm{X}_{\mathrm{W}}\right)$ between the LTE eNB and WLAN, as shown in Figure 7. In LWA, the WLAN radio link behaves as secondary access for the user data, while the signalling 


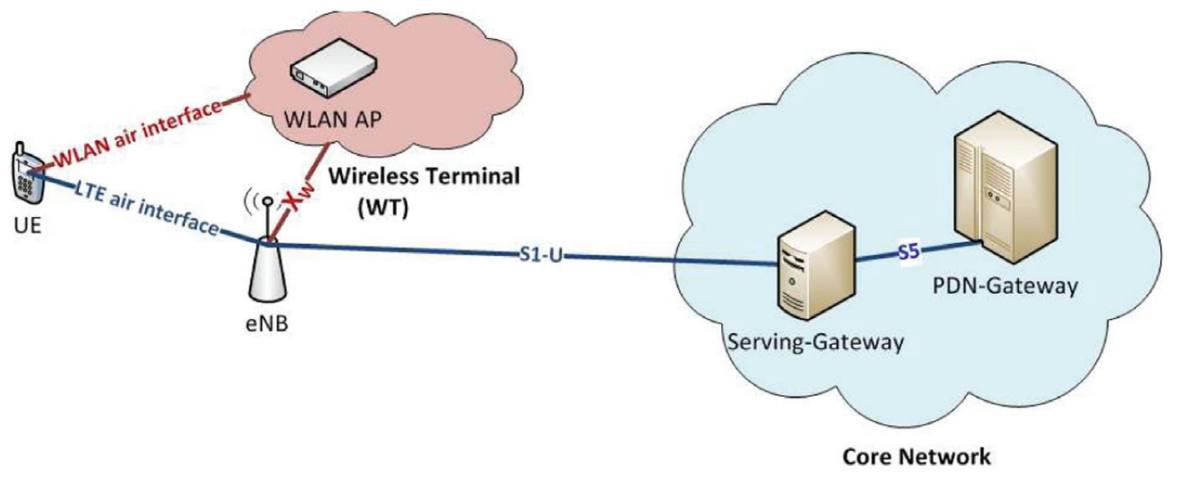

Figure 7 LWA architecture.

related to LWA is managed by eNB via RRC layer. The user plane PDCP Protocol Data Unit (PDU) is delivered by either the LTE or the WLAN radio link. 3GPP introduced a new LTE-WLAN Aggregation Adaptation Protocol (LWAAP) to handle the user plane data between the PDCP and the WLAN MAC and also to identify the LWAAP Service Data Unit (SDU) to the corresponding LWA bearer [22]. Further two types of bearers, split LWA bearer and switched LWA bearer, are supported for the LWA, as shown in Figure 8.

For the LTE-WLAN Aggregation, the end-points of security protection (encryption/decryption) remain at the respective PDCP layers of the eNB and the UE, even though the PDCP packets traverse via the non-native path (via the WLAN Termination (WT)). In addition to the PDCP layer protection, the WLAN communication established between the WT and the UE shall be protected using the native IEEE 802.11i [18] security mechanisms. The security key for protecting the WLAN link is computed from the current LTE Access Stratum (AS) security context established between the UE and the eNB. When the eNB initially establishes LWA with the UE through a WT, the LTE AS security context shared between the eNB and the particular UE is used by the eNB to generates the key S-KWT for the WT and sends it to the WT over the Xw. The same S-KWT is generated by the UE using its current LTE AS security context, thus the UE and the WT have the same key. The UE and the WT use the key S-KWT as the Pair Wise Master Key (PMK) defined in IEEE 802.11 specification [18] to establish the WLAN security. The PMK will be used to start the 4-way handshake on the WLAN link between the UE and the WLAN AP. 


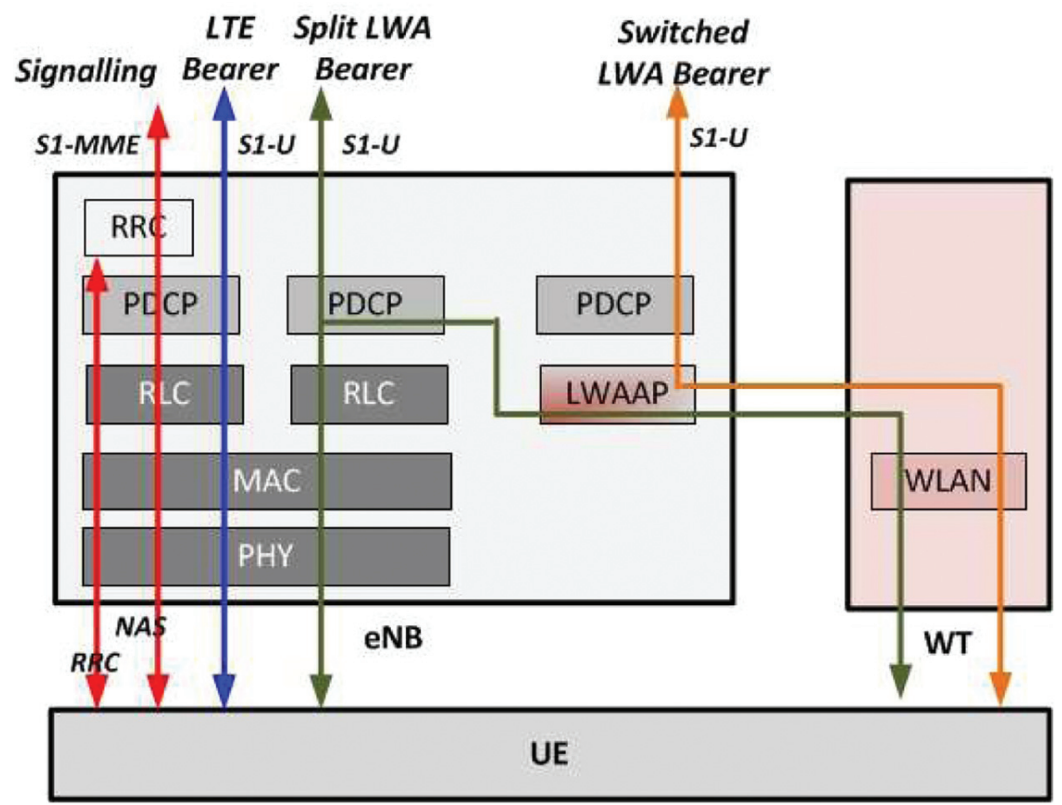

Figure 8 LWA protocol stack.

\subsubsection{LTE-WLAN radio level integration with IPsec tunnel (LWIP)}

PDCP aggregation may be supported with the legacy WLAN Access Points together with non-collocated LTE eNBs provided a link exists between them for the WLAN Access Points to report information such as load information and Modulation and Coding Scheme (MCS) to the eNB. Operators interested in deploying this feature do not have to enhance or modify their existing WLAN infrastructure.

LWA RAN level solution, with RAN level routing and routing decision, is extended for the legacy (non-co-located) WLAN deployment scenarios also. 3GPP defined a solution for RAN based LTE-WLAN aggregation at a bearer level which addresses the legacy WLAN deployment scenarios. For the legacy scenarios, the LTE-WLAN integration is accomplished by using IPSec tunnelling [9] for the PDCP SDUs above the PDCP layer. The eNB controls activation of the integration based on the UE connectivity with a specific WLAN. Once the integration is activated, the eNB segregates incoming downlink packets towards the UE for offloading via the WLAN at a layer above PDCP as shown in Figure 9. The uplink packets from the 


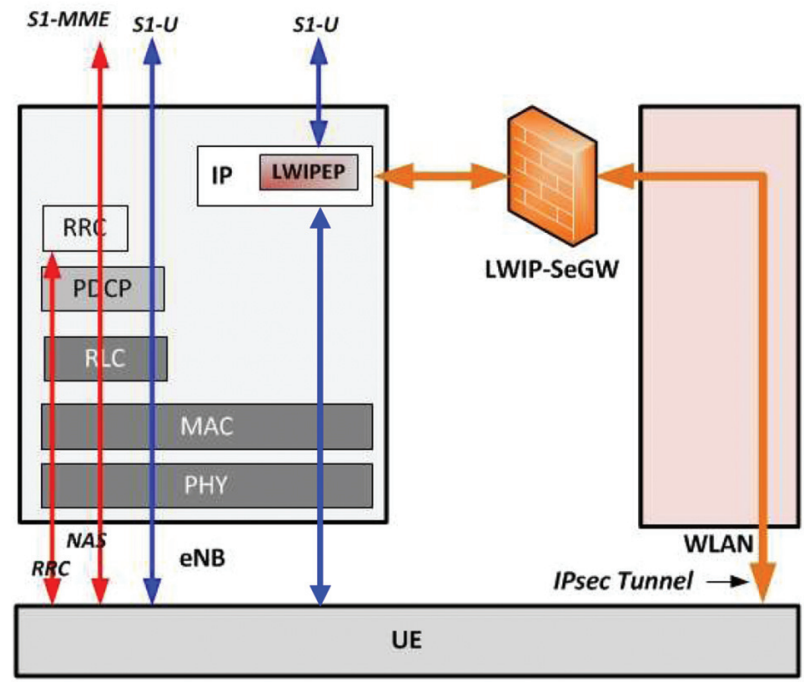

Figure 9 LWIP architecture.

UE are aggregated by the eNB at the same logical point. 3GPP specified the LWIP Encapsulation Protocol (LWIPEP) for transfer of user plane data and also identification of the LWIPEP SDU to map to the corresponding the data bearer identity [23].

LTE-WLAN integration (LWIP) over legacy WLAN is secured using an IPSec [9] in a tunnel mode [10] established between the UE, via the WLAN, and the LWIP-SeGW function. 3GPP introduce LWIP SeGW for terminating IPsec tunnel from the UE, as to mitigate the threats on the eNB, as the eNB is accessible via the IP interface for the LWIP. The IPSec in tunnel mode is established using the IKEv2 handshake based on the pre-shared key (PSK) [19]. The UE and LWIP-SeGW use the LWIP-PSK as the PSK for authentication in the second phase of IKEv2 [20]. Signalling bearers are carried over the LTE only.

\section{Summary and Future Work}

This paper presented the evolution of 3GPP systems (3G-UMTS, LTE and LTE-A) interworking with the WLAN from Release- 6 to Release-13. The paper presents the Inter-RAT mobility modes, access network selection procedures and RAN level integration between the 3GPP systems and the WLAN in detail. Efficient interworking with the WLAN will be the vital components 
of the next generation cellular wireless networks. Interworking between the $3 \mathrm{GPP}$ systems and the WLAN will continue to be improved, leading to a tighter interworking approach and seamless simpler mobility. With the spreading use of the WLAN and the increasing role WLAN is playing in the 3GPP operator network deployments, improving the interworking of the WLAN with the EPC would be beneficial to the operators and to the user experience.

3GPP is still searching the direction, which to consider, in terms of level of integration with the WLAN, ranging between the reasonable enhancements in the 3GPP systems for the WLAN selection parameters up to carrier aggregation with the WLAN. With the increased mobile data usage and wireless data rates, coupled with more widespread home and public WLAN networks, the relevance of offloading mechanisms will continue in the upcoming 3GPP Releases and will be one of the key areas of innovation within the 3GPP forum. To cost-effectively leverage the unlicensed radio access technology with the strategy of revenue maximization, the WLAN will be more tightly integrated with the 3GPP RATs, beside with the required level of security and acceptable QoS provision, in the future.

\section{References}

[1] 3GPP Forum. Available at: http://www.3GPP.org/

[2] 3GPP. Requirements on 3GPP system to Wireless Local Area Network (WLAN) interworking, 3GPP TS 22.234.

[3] 3GPP. 3GPP system to Wireless Local Area Network (WLAN) Interworking, System Description, 3GPP TS 23.234.

[4] 3GPP. Service requirements for the Evolved Packet System (EPS), 3GPP TS 22.278

[5] 3GPP. Architecture enhancements for non-3GPP accesses, 3GPP TS 23.402.

[6] 3GPP. LS on ANDSF Enhancements, SA2 WG, 3GPP S2-087346.

[7] 3GPP. Evolved Universal Terrestrial Radio Access (E-UTRA) and Evolved Universal Terrestrial Radio Access Network (E-UTRAN); Overall description; Stage 2, 3GPP TS 36.300.

[8] 3GPP. 3G Security, Interworking Security, 3GPP TS 33.234.

[9] Kent, S., and Atkinson, R. (1998). Security Architecture for the Internet Protocol, IETF RFC 2401.

[10] Kent, S., and Atkinson, R. (1998). IP Encapsulating Security Payload (ESP), IETF RFC 2406. 
[11] Rajavelsamy, R., Jeedigunta, V., Holur, B., Choudhary, M., and Song O. (2005). "Performance Evaluation of VoIP over 3G-WLAN Interworking System," in Proceedings of IEEE Wireless Communication and Networking Conference, Vol. 4, 2312-2317.

[12] 3GPP. 3G security; Wireless Local Area Network (WLAN) interworking security, 3GPP TS 33.234.

[13] 3GPP. 3GPP System Architecture Evolution (SAE); Security aspects of non-3GPP accesses, 3GPP TS 33.402.

[14] 3GPP. Service Requirements for Evolution of the 3GPP System, 3GPP TS 22.278.

[15] 3GPP. IP flow mobility and seamless Wireless Local Area Network (WLAN) offload; Stage 2, 3GPP TS 23.261.

[16] Wi-Fi Hotspot 2.0 Technical Specification R2 V4.00

[17] Arkko, et al. (2009). Improved Extensible Authentication Protocol Method for 3rd Generation Authentication and Key Agreement (EAP$\left.A K A^{\prime}\right)$, IETF RFC 5448.

[18] IEEE Std. 802.11i. (2004). Telecommunications and information exchange between systems- Local and metropolitan area networksSpecific requirements Part 11: Wireless LAN Medium Access Control (MAC) and Physical Layer (PHY) specifications Amendment 6: Medium Access Control (MAC) Security Enhancements.

[19] Kaufman, et al. (2014). Internet Key Exchange Protocol Version 2 (IKEv2), RFC 7296.

[20] Eronen and Korhonen. (2006). Multiple Authentication Exchanges in the Internet Key Exchange (IKEv2) Protocol, IETF RFC 4739.

[21] 3GPP. 3GPP system - fixed broadband access network interworking, 3GPP TS 23.139.

[22] 3GPP. LTE-WLAN Aggregation Adaptation Protocol, 3GPP TS 36.360.

[23] 3GPP. LTE/WLAN Radio Level Integration Using IPsec Tunnel (LWIP) encapsulation; Protocol specification, 3GPP TS 00.001.

[24] 3GPP. General Packet Radio Service (GPRS) enhancements for Evolved Universal Terrestrial Radio Access Network (E-UTRAN) access, 3GPP TS 23.401. 


\section{Biographies}

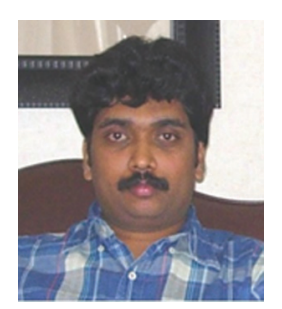

Rajavelsamy $\mathbf{R}$ is a group engineering manager (principal engineer) in the Advanced Research and Standards Division of Samsung R\&D Institute India, Bangalore, focusing on research and standardization activities for next generation mobile communication security aspects (LTE, LTE-A, LTE-WLAN interworking, HeNB, Machine Type Communications, Small Cell Enhancements (SCE), Proximity Services (ProSe), Mission Critical communications) and has publications in these areas. He received his B.E. degree in electronics and communication engineering from Bharathiar University, India, in 1999. He worked as a project engineer at the Indian Institute of Science from 1999 to 2003. His area of work is wireless networks, security, and IP technologies. He joined Samsung Electronics at its Bangalore office in 2003. Since 2004, he has been actively participating and contributing to 3GPP SA3 Security Working Group, where he served as Vice Chairman (2007-2009) and Rapporteur of Machine Type Communication (TS 33.187, TR 33.868, TR 33.889) work items.

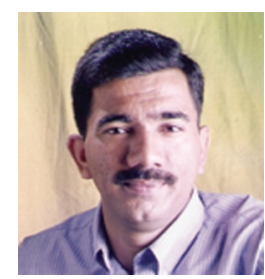

M. Choudhary is currently the Director of Research Strategy at Samsung R\&D Institute India, Bangalore. Prior to this assignment, he was the Director of Digital Media \& Communication (DMC R\&D) and SAIT (Samsung Advanced Institute of Technology) India at Bangalore for 5 years.

He received his Ph.D from IIT Kanpur. Prior to Samsung, he worked with Texas Instruments (India) as Development Unit Manager of modem software development for mobile handsets. 
His earlier work at Samsung has been towards research and standardization of emerging wireless technologies such as Ultra Wide Band and 3G-WLAN interworking. He has been one of the authors of Ultra Wide Band MAC specification at the WiMedia Alliance and also served as co-chair of Technical Steering Committee for the WiMedia Alliance.

Dr. Manoj has delivered numerous talks and tutorials in national/international conferences and industry forums. He has 20 granted patents (and 10+ pending applications) in US, Korea, European Union and India. Dr. Manoj is also co-author of the book "Ultra Wideband Demystified: Technologies, Applications, and System Design Considerations" published by River Publishers, Denmark. He is a Senior Member of the IEEE.

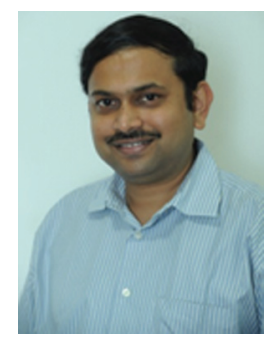

D. Das is serving as Dean - Academic and R\&D as well as Hewlett Packard Chair Professor at IIIT-Bangalore (IIIT-B). Before joining IIIT-B, he had served at G S Sanyal School of Telecommunication at IIT Kharagpur and later at Kirana Networks in New Jersey, USA. At present, he is Principal Investigator (PI) of projects from Department of Electronics and Information Technology, Government of India on Green Broadband Wireless Network and Nokia Research on IoT. He was PI of sponsored projects from Intel, Hewlett Packard, Microsoft, Motorola Research, Nokia, Govt. of India on areas of IMS and Broadband Wireless MAC/QoS/Energy-saving, TVWS. He has more than 85 peer reviewed papers in different journals and International conferences. His 7 patents are under process. Dr. Das received his Ph.D. degree from IIT-Kharagpur and MTech from IIT-Varanasi. He is Board Member of IIIT-Bhubaneswar; Technical and Empower Committee member of e-Governance, Govt. of Karnataka; He is Fellow of Institution of Electronics\& Telecommunication Engineers (IETE). Dr. Das is recipient of Global IEEE MGA Achievement Award 2012. 